\title{
A new area editor
}

\author{
Masayasu Mimura
}

Published online: 23 April 2014

(C) The JJIAM Publishing Committee and Springer Japan 2014

We have been receiving tremendous benefits due to innovations in science, engineering, and technology, which have been developing exponentially since the last century. However, at the same time, complexity and its significance have been growing drastically in many systems: for example, engineered systems such as the Internet and the power grid, social systems such as the financial system and social networks, and natural systems such as the global climate system and the global ecological system. We should note that, uncertainty and risk are integral parts of these complex systems and they have become more important.

Considering this situation, JJIAM has opened a new area 4, which focuses on the following fields: probabilistic and statistical methodology, financial mathematics, insurance mathematics, stochastic control, stochastic numerics, and related modeling. Consequently, we have decided to add a new area editor.

I am pleased to announce an important expansion of the editorial board: Dr. Jun Sekine has been invited as the new area editor. Professor Sekine is a faculty member of Mathematical Sciences for Social Systems, Department of Systems Innovation, Graduate School of Engineering Science, Osaka University.

I would like to thank him and all our current area editors for their efforts on behalf of JJIAM.

Masayasu Mimura

Editor-in-Chief, Japan Journal of Industrial and Applied Mathematics

M. Mimura ( $\square)$

Meiji Institute for Advanced Study of Mathematical Sciences,

Meiji University, 4-21-1 Nakano, Nakano-ku, Tokyo 164-8525, Japan

e-mail: mimura.masayasu@gmail.com 